\title{
Baustelle Grundversorgung: Notfalldienst und Praxisassistenz
}

Ende März 2006 beschlossen das Departement des Innern (EDI) und die Gesundheitsdirektorenkonferenz (GDK) aufgrund eines Arbeitspapiers [1] zur Unterstützung der Grundversorgung, eine Arbeitsgruppe unter Federführung der GDK einzuberufen. In zwei Untergruppen erarbeiteten Vertreterinnen und Vertreter von kantonsärztlichen Diensten, kantonalen Ärztegesellschaften, KHM, VSAO, FMH, santésuisse, BAG und GDK Vorschläge zur Behebung der Engpässe im ambulanten ärztlichen Notfalldienst und zur Finanzierung von spezifischen Weiterbildungsangeboten in der Hausarztmedizin. Die Arbeitsgruppe verabschiedete am 19. September 2006 die Berichte, welche durch das Sekretariat der GDK redigiert und Ende Oktober den politischen Entscheidungsträgern vorgelegt werden sollen.

Im Bereich des ärztlichen Notfalldienstes brachten diese Arbeiten in der ganzen Schweiz einen Prozess in Gang, welcher die ärztliche Notfallversorgung der Bevölkerung sichern wird und gleichzeitig effizienter werden lässt. In den sehr unterschiedlich grossen Versorgungsregionen mit ebenso unterschiedlicher Ärztedichte wird nach partnerschaftlichen Lösungen zwischen ambulanten und stationären Leistungserbringern gesucht.

Im Bereich der ärztlichen Weiterbildung in der Grundversorgung lösten die Arbeiten, noch vor Verabschiedung des Berichtes, interessante kantonale und regionale Aktivitäten aus. Die Bedeutung der Weiterbildung im künftigen Arbeitsumfeld ist allgemein erkannt. Kurzfristig soll die Praxisassistenz durch finanzielle Unterstützung der
Kantone der Assistenz in Spitälern gleichgestellt werden. Mittelfristig müssen Bedingungen geschaffen werden, damit sich ein gut funktionierender Weiterbildungsmarkt entwickeln und die Weiterbildung der künftigen Grundversorgerinnen und Grundversorger effizienter gestaltet werden kann.

Die Bauarbeiten gehen also weiter. Die Expertinnen und Experten der Arbeitsgruppe wurden von der GDK erneut einberufen: Jedes einigermassen effiziente Gesundheitssystem benötigt eine qualitativ und quantitativ breite Grundversorgung [2]. Die Aus- und Weiterbildung der künftigen Grundversorgerinnen und Grundversorger muss nach dem Bedarf der Bevölkerung [3] ausgestaltet, für das Zeitalter der prädiktiven Medizin gestärkt und effizienter werden. Die Tätigkeit in der Grundversorgung muss attraktiver und den sozialen und demographischen Verhältnissen der künftigen Ärztegeneration angepasst werden.

Max Giger, Mitglied des Zentralvorstands der FMH, Präsident der Kommission für Weiterund Fortbildung der FMH

1 Trutmann M. Magna cum cura. www.gdk-cds.ch/ fileadmin/pdf/Gesundheitsversorgung/Planung Gesversorgung/Grundversorgung/Grundversorger_III.pdf.

2 Starfield B, Shi Leiyo, Macinko J. Contribution of primary care to health systems and health. Milbank Quarterly. 2005; 83:457-502.

3 Safran DG. Defining the future of primary care: what can we learn from patients? Ann Intern Med. 2003;138:248-55.

Hinweis: Der in diesem Editorial erwähnte Bericht wird von der GDK bald veröffentlicht und in Form eines zusammenfassenden Artikels der SÄZ publiziert - bei dieser Gelegenheit werden wir das Thema dann also erneut und auch von anderer Seite beleuchten. 